See discussions, stats, and author profiles for this publication at:

http://www.researchgate.net/publication/260950751

\title{
The effects of elastic tubing-based resistance training compared with conventional resistance training in patients with moderate chronic obstructive pulmonary disease: a randomized...
}

ARTICLE in CLINICAL REHABILITATION · MARCH 2014

Impact Factor: 2.24 · DOI: 10.1177/0269215514527842 · Source: PubMed

CITATIONS

2

11 AUTHORS, INCLUDING:

\section{Alessandra Toledo-Arruda}

Universidade Federal Fluminense

45 PUBLICATIONS 218 CITATIONS

SEE PROFILE
DOWNLOADS

158
VIEWS

166
Rubens Cecchini

Universidade Estadual de Londrina

85 PUBLICATIONS 872 CITATIONS

SEE PROFILE 


\section{Clinical Rehabilitation}

http://cre.sagepub.com/

The effects of elastic tubing-based resistance training compared with conventional resistance training in patients with moderate chronic obstructive pulmonary disease: a randomized clinical trial

Ercy Mara Cipulo Ramos, Alessandra Choqueta de Toledo-Arruda, Luciana Cristina Fosco, Rafaela Bonfim, Giovana Navarro Bertolini, Flavia Alessandra Guarnier, Rubens Cecchini, Carlos Marcelo Pastre, Daniel

Langer, Rik Gosselink and Dionei Ramos

Clin Rehabil published online 19 March 2014

DOI: $10.1177 / 0269215514527842$

The online version of this article can be found at:

http://cre.sagepub.com/content/early/2014/03/18/0269215514527842

Published by:

(SAGE

http://www.sagepublications.com

Additional services and information for Clinical Rehabilitation can be found at:

Email Alerts: http://cre.sagepub.com/cgi/alerts

Subscriptions: http://cre.sagepub.com/subscriptions

Reprints: http://www.sagepub.com/journalsReprints.nav

Permissions: http://www.sagepub.com/journalsPermissions.nav

>> OnlineFirst Version of Record - Mar 19, 2014

What is This? 


\section{The effects of elastic tubing-based resistance training compared with conventional resistance training in patients with moderate chronic obstructive pulmonary disease: a randomized clinical trial}

Clinical Rehabilitation

I-II

(C) The Author(s) 2014

Reprints and permissions:

sagepub.co.uk/journalsPermissions.nav DOI: $10.1177 / 0269215514527842$ cre.sagepub.com

(S)SAGE

\author{
Ercy Mara Cipulo Ramos', Alessandra Choqueta de \\ Toledo-Arruda ${ }^{1,2}$, Luciana Cristina Fosco', Rafaela \\ Bonfim', Giovana Navarro Bertolini', Flavia Alessandra \\ Guarnier ${ }^{3}$, Rubens Cecchini', Carlos Marcelo Pastre', \\ Daniel Langer ${ }^{4}$, Rik Gosselink ${ }^{4}$ and Dionei Ramos'
}

\author{
Corresponding author: \\ Ercy Mara Cipulo Ramos, Departamento de Fisioterapia, \\ Universidade Estadual Paulista, Rua Roberto Simonsen, No. \\ 305, Presidente Prudente, Sao Paulo 19060-900, Brazil. \\ Email: ercy@fct.unesp.br
}


and last sessions, there was an increase in interleukin I $\beta(\mathrm{IL}-\mathrm{I} \beta)$ and interleukin I0 (IL-I0) in both groups, while tumour necrosis factor alpha (TNF- $\alpha$ ) was stimulated only in the conventional training group.

Conclusion: Elastic tubing training had a greater effect on functional exercise capacity than conventional resistance training. Both interventions were equally effective in improving muscle strength and quality of life.

\section{Keywords}

Chronic obstructive pulmonary disease, muscle strength, rehabilitation, resistance training

Received: 3 March 2013; accepted: 22 February 2014

\section{Introduction}

Unlike aerobic training, resistance training in patients with chronic obstructive pulmonary disease enables exercise to be performed with a smaller muscle mass, which yields a higher metabolic rate because the respiratory system is subjected to less stress compared with whole-body exercises. ${ }^{1}$ Evidence supports the use of peripheral resistance training as a means of improving functional and exercise capacity, dyspnoea, peripheral muscle strength, ambulation, fat-free mass, and quality of life in patients with chronic obstructive pulmonary disease..$^{2-6}$ Although peripheral resistance training in elderly patients can reduce the levels of inflammatory mediators and the deleterious consequences of these cytokines on muscle loss, ${ }^{7-9}$ these findings are inconclusive in patients with chronic obstructive pulmonary disease. ${ }^{10}$

Weight machines are the conventional devices used in clinical studies and practice for resistance training interventions in patients with chronic obstructive pulmonary disease. A few previous studies also demonstrated the positive effects of elastic tubing in this population. ${ }^{11-13}$ Elastic tubing exercises are performed with one leg/arm at a time, and they increase the total amount of work performed and local muscle endurance compared with the results obtained from training both limbs. ${ }^{14,15}$ However, the effectiveness of elastic tubing-based resistance training compared with conventional resistance training (incorporating upper as well as lower extremity muscles) has not yet been investigated.

The present study was designed to compare the effects of peripheral resistance training performed with elastic tubing and a conventional resistance training intervention using weight machines on functional exercise capacity, muscle strength, fatfree mass, and cytokine profile in patients with chronic obstructive pulmonary disease.

\section{Methods}

\section{Participants}

This randomized clinical trial was conducted in a university-based, outpatient, physical therapy clinic in compliance with the Declaration of Helsinki. The criteria for participant selection were the following: (1) patients had to have a diagnosis of chronic obstructive pulmonary disease according to the Global Initiative for Chronic Obstructive Lung Disease (GOLD) ${ }^{16}$ and (2) patients had to be clinically stable with no exacerbations or changes in medication for at least 30 days. None of the individuals were current smokers, used oxygen therapy at home, or were engaged in any exercise training programme before participating in this study. The exclusion criteria were as follows: (1) disabling pathological conditions that could potentially influence physical activity performance, such as cerebrovascular diseases, rheumatism, and arthritis; (2) unstable chronic obstructive pulmonary disease; (3) severe and/or unstable cardiac disease as revealed by electrocardiogram; and (4) other pathological conditions that could influence the systemic inflammatory process. All patients remained stable during the eight-week assessment period. 
The study was approved by the institutional review board, and all individuals signed an informed consent form before participating in the study.

A total of 45 stable chronic obstructive pulmonary disease patients were included in the study. The patients were randomly allocated to the following two groups by drawing lots: conventional training $(\mathrm{CT})(n=23)$ and elastic tubing training (ETT) $(n=22)$ groups. Anonymity was preserved by allocating a number to each patient before the drawing. Random assignment was achieved by an independent person who drew the sealed numbers before the start of the intervention. The participants were blinded to the study hypotheses.

Intervention. The eight-week resistance training programme consisted of 60-minute training sessions performed three times per week. Each of the 24 sessions consisted of a warm-up at the beginning and general stretching at the end. Resistance exercises for knee extension and flexion, shoulder abduction and flexion, and elbow flexion were performed using two devices: elastic tubing (Lemgruber ${ }^{\circledR}$ brand, Brazil) for the elastic tubing group and weight machines (Ipiranga ${ }^{\circledR}$ Gym line, Brazil) for the conventional group. Three senior physical therapists specialised in pulmonary rehabilitation and/ or musculoskeletal rehabilitation and four physical therapists were responsible for the assessments and holding the exercise sessions with both intervention groups. The physiotherapists provided close supervision during every session for both training protocols. The researcher who performed the data analyses was blinded to group allocation throughout the study.

Commercially available elastic tubing (Lemgruber ${ }^{\circledR}$ brand, Brazil) with internal diameters of $4 \mathrm{~mm}$ and $6 \mathrm{~mm}$ and external diameters of $8 \mathrm{~mm}$ and $12 \mathrm{~mm}$ for the upper and lower limbs, respectively, was used in the ETT group. The total cost of these materials was approximately $\$ 20$. The elastic tubing group was trained after assessing the individual's repetition variability, as determined by repeated administration of the fatigue resistance test with one-minute rest intervals. The fatigue resistance test aimed to provoke task failure owing to fatigue 40-60 seconds after the beginning of each movement execution (knee extension and flexion, shoulder abduction and flexion, and elbow flexion). During the test, the patients were instructed to perform each movement with a full range of motion for the maximum number of repetitions and at maximum speed while free from signs or symptoms. The test was interrupted in the case of fatigue, significant reduction in the range of motion and speed, or muscle compensation. Execution times greater or less than 40-60 seconds indicated the need to perform a new test. The execution time was used as a reference to obtain the number of repetitions proportional to 20 seconds, the duration for which the adenosine triphosphatephosphocreatine (ATP-PC) system was used by muscle cells to obtain energy. We recorded the number of repetitions and the time until exhaustion (from 40 to 60 seconds). The elastic tubing group was trained by performing two to seven individual sets, and each set consisted of the number of repetitions achieved during 20 seconds of the fatigue resistance test. The load was increased by adding a set every two sessions. The subjects had a twominute rest period between each set. This training protocol was based on our clinical experience. After four weeks, the fatigue resistance test was repeated to determine the new number of required repetitions for the next four weeks.

The conventional method of resistance training used in our study was most commonly found in previous studies in patients with chronic obstructive pulmonary disease. ${ }^{17-19}$ The conventional group started at $60 \%$ of their one-repetition maximum (1-RM; the maximum load that can be moved only once over the full range of motion without compensatory movements), and they performed three sets of 10 repetitions for each exercise. The load was increased by $4 \%$ every four sessions until they reached $80 \%$ of their initial 1-RM at the end of the protocol.

Outcome measures. Lung function measurements were obtained for all subjects at baseline. Spirometry was performed according to the guidelines of the American Thoracic Society and European Respiratory Society ${ }^{20}$ using a portable spirometer 
(Spirobank-MIR, Italy, version 3.6). The reference values were specific for the Brazilian population. ${ }^{21}$

The primary outcome measure was the functional exercise capacity at baseline and after eight weeks of training. Peripheral muscle strength, quality of life, and fat-free mass were measured at the same time points. The cytokine profile was measured before and after the first and last training sessions.

The 6-minute walk test was the primary outcome measure because it assesses functional exercise capacity according to the guidelines of the American Thoracic Society. ${ }^{22}$ The experimental values obtained were referenced to normal values provided by Enright and Sherrill. ${ }^{23}$

Peripheral muscle strength tests of knee flexion and extension, shoulder flexion and abduction, and elbow flexion were performed unilaterally (dominant limb) with a digital dynamometer (Force Gauge $^{\circledR}$ brand, FG $-100 \mathrm{~kg}$ ), and the results are expressed in newtons $(\mathrm{N})$. The volunteers performed a maximal voluntary isometric contraction for six seconds followed by limb relaxation. The measurement was repeated three times with rest intervals of one minute, and the highest value was recorded.

Health-related quality of life was assessed by the Chronic Respiratory Disease Questionnaire (CRDQ), which is a four-domain questionnaire with 20 questions divided into four domains: dyspnoea (five questions), fatigue (four questions), emotional function (seven questions), and mastery (four questions). The score of each question varies according to a scale from one (maximum impairment) to seven (no impairment). ${ }^{24}$ The improvement or deterioration in quality of life was considered to be clinically important for differences greater than 0.5 points. ${ }^{25}$

The fat-free mass was assessed by dual-energy X-ray absorptiometry (DXA; Hologic model QDR machine dials 2000/Plus - Hologic, Waltham; Software version 5.56). ${ }^{26}$ Of the 34 volunteers who completed the experimental protocol, 24 underwent this evaluation (12 patients in each group).

Cytokines were quantified in plasma samples taken at baseline, immediately after the first session (acute response to exercise), before the last training session (after eight weeks of training), and immediately after the last training session (acute response to exercise after training). Tumour necrosis factor alpha (TNF- $\alpha$ ), interleukin $1 \beta$ (IL- $1 \beta)$, and interleukin 10 (IL-10) were measured in the plasma by an enzyme-linked immunosorbent assay (ELISA). All blood samples were drawn in the morning, between 8 and 10 a.m., and were immediately separated by centrifugation at $3800 \mathrm{r} / \mathrm{min}$ for 10 minutes at $4{ }^{\circ} \mathrm{C}$. Aliquots of the plasma were stored at $-70{ }^{\circ} \mathrm{C}$ for subsequent assays. The plasma concentrations were measured by ELISA using a commercial kit for TNF- $\alpha$, IL-1 $\beta$, and IL-10 (eBioscience, San Diego, CA, USA) and read on a microplate reader (Biotek, Biosystems, Winooski, VT, USA). The readings were obtained at a wavelength of $490 \mathrm{~nm}$, and the results are expressed in $\mathrm{pg} / \mathrm{mL}$ of plasma.

The sample size required for this study was based on a preliminary study. ${ }^{18}$ Our analysis indicated that a sample size of 40 participants $(20$ per group) was needed to detect a minimal clinically important difference of 54 meters with $80 \%$ power for the functional exercise capacity ${ }^{27}$ outcome in both groups. We anticipated that approximately $20 \%$ of the participants may fail the initial screening or drop out; therefore, we needed to enrol 48 participants to account for this loss.

Data analysis. The data normality was evaluated using the Shapiro-Wilk test. The baseline results are presented as the means and standard deviation (SD). Baseline differences between groups were evaluated with the chi-square test for categorical data (gender), and Student's $t$-test was used for continuous data. Differences between the two groups in the 6-minute walk test, peripheral muscle strength, and fat-free mass at baseline and after eight weeks of training were analysed with a twotailed unpaired $t$-test. The CRDQ data were assessed by the Mann-Whitney test, and the data were presented as medians with $95 \%$ confidence intervals (CIs). The cytokine levels were analysed with a two-way repeated-measures analysis of variance (ANOVA). A linear correlation analysis was performed with the Pearson test. Differences were considered significant at $P<0.05$. 


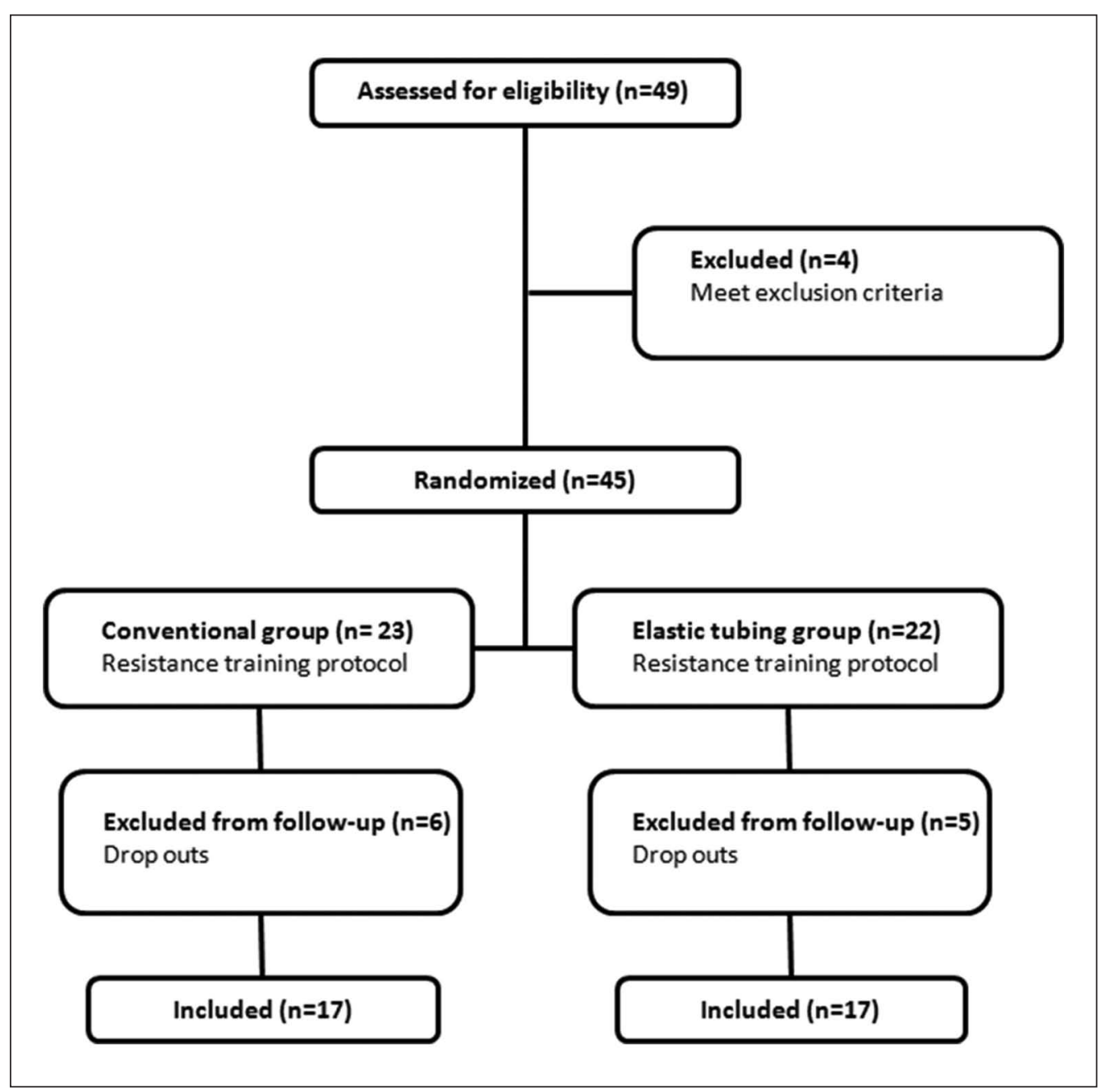

Figure I. Study flowchart.

All statistical analyses were performed using SPSS software version 16 (SPSS, Inc., Chicago, Illinois, USA).

\section{Results}

A total of 49 patients were screened, and four patients were excluded owing to musculoskeletal diseases that would impair exercise training. During the programme, 11 patients dropped out of the study (CT, $n=6$; ETT, $n=5$ ) owing to reasons unrelated to disease or treatment. Figure 1 shows the study flow of this randomized controlled trial.
At baseline, both groups were similar with respect to age, gender, BMI, lung function, health-related quality of life, functional exercise capacity, and fatfree mass. The baseline characteristics are summarised in Table 1 . There was a positive, moderate linear correlation between the 6-minute walking distance at baseline and the knee extension strength at baseline in the CT and ETT groups $(r=0.44$ and $r=0.61$, respectively). On average, patients showed moderate airflow obstruction, normal BMI, and similar plasma cytokine levels at baseline in both groups. Only TNF- $\alpha$ was significantly higher in the ETT group. 
Table I. Baseline characteristics of COPD patients.

\begin{tabular}{lcc}
\hline & $\begin{array}{c}\text { Conventional resistance } \\
\text { training }(n=17)\end{array}$ & $\begin{array}{c}\text { Elastic tubing training } \\
(n=17)\end{array}$ \\
\hline Age $(\mathrm{yr})$ & $66(6 \mathrm{I}-68)$ & $67(60-69)$ \\
Gender $(\mathrm{M} / \mathrm{F})$ & $13 / 4$ & $1 \mathrm{I})$ \\
$\mathrm{FFMI}\left(\mathrm{kg} / \mathrm{m}^{2}\right)$ & $16(15-17.5)$ & $15.5(14.5-17)$ \\
FEV $(\mathrm{L})$ & $1.3(1-1.4)$ & $1.1(1-1.5)$ \\
FVC $(\mathrm{L})$ & $2.4(1.8-2.7)$ & $2.3(2.1-3)$ \\
FEVI/FVC $(\%)$ & $53.6(49.1-58.5)$ & $49.5(43.5-55.4)$ \\
6MWD $(\%$ Pred) & $85(76-95)$ & $84(68-92)$ \\
CRDQ total score & $91(74-97)$ & $82(76-92)$ \\
\hline
\end{tabular}

Data are expressed as the median with the $25-75 \%$ confidence interval. The gender data are expressed as the number of subjects. M: male; F: female; FFMI: fat-free mass index; FEV : forced expiratory volume in I second; FVC: forced vital capacity; 6MWD: 6-minute walk distance; CRDQ: Chronic Respiratory Disease Questionnaire.

Following the completion of both protocols, there was an increase in peripheral muscle strength in both groups $(P<0.05)$. These improvements were not significantly different between groups (Table 2).

The performance in the 6-minute walk test improved after eight weeks of training in both groups (Table $2, P<0.001$ ), and this increase was larger in the elastic tubing group compared with the conventional training group $(P<0.05)$.

The health-related quality of life improved according to the total CRDQ score in both groups, with no difference between the groups $(P<0.05)$. The specific CRDQ domains revealed significant improvements in dyspnoea and mastery $(P<0.05)$ in the CT group and dyspnoea and emotional function in the ETT group $(P<0.05)$. In the ETT group, all domains reached the minimum clinically important difference after eight weeks of training (dyspnoea, 0.9; fatigue, 0.7; emotional function, 0.8; mastery, 0.5), whereas in the CT group the emotional function and fatigue domains did not reach the 0.5-point difference after the training (dyspnoea, 1.0; fatigue 0.3; emotional function, 0.4 ; mastery, 0.7). A positive, moderate linear correlation was observed between the improvement in fatigue and improvement in dyspnoea in the CT and ETT groups $(r=0.46$ and $r=0.52$, respectively).

The fat-free mass was assessed in 24 patients ( $n$ $=12$ in each group). After training, there was a trend toward improvement in the total fat-free mass in both groups (Table 2, $P=0.05$ ). There were no differences between the groups with respect to fatfree mass in either the leg or arm. The plasma cytokine profiles in both groups at baseline, immediately after the first session (acute response to exercise), before the last training session (after eight weeks of training), and immediately after the last training session (acute response to exercise after eight weeks of training) are presented in Table 3. There was a negative, weak correlation between the expression of TNF- $\alpha$ at baseline and improvement in the 6-minute walking distance after eight weeks of training only in the ETT group $(r=-0.35$ and $r=-0.002$ for the ETT and CT groups, respectively).

\section{Discussion}

The present study showed that a new protocol of resistance training using elastic tubing results in equivalent improvements in quality of life and muscle strength and has larger effects on functional exercise capacity compared with $\mathrm{CT}$. Fat-free mass remained constant after training despite changes observed in inflammatory cytokines.

Elastic tubing is an easy-to-use, inexpensive device and is accessible to a greater number of chronic obstructive pulmonary disease patients. It also allows patients to perform exercises at home. However, there are limited studies on the effects of 


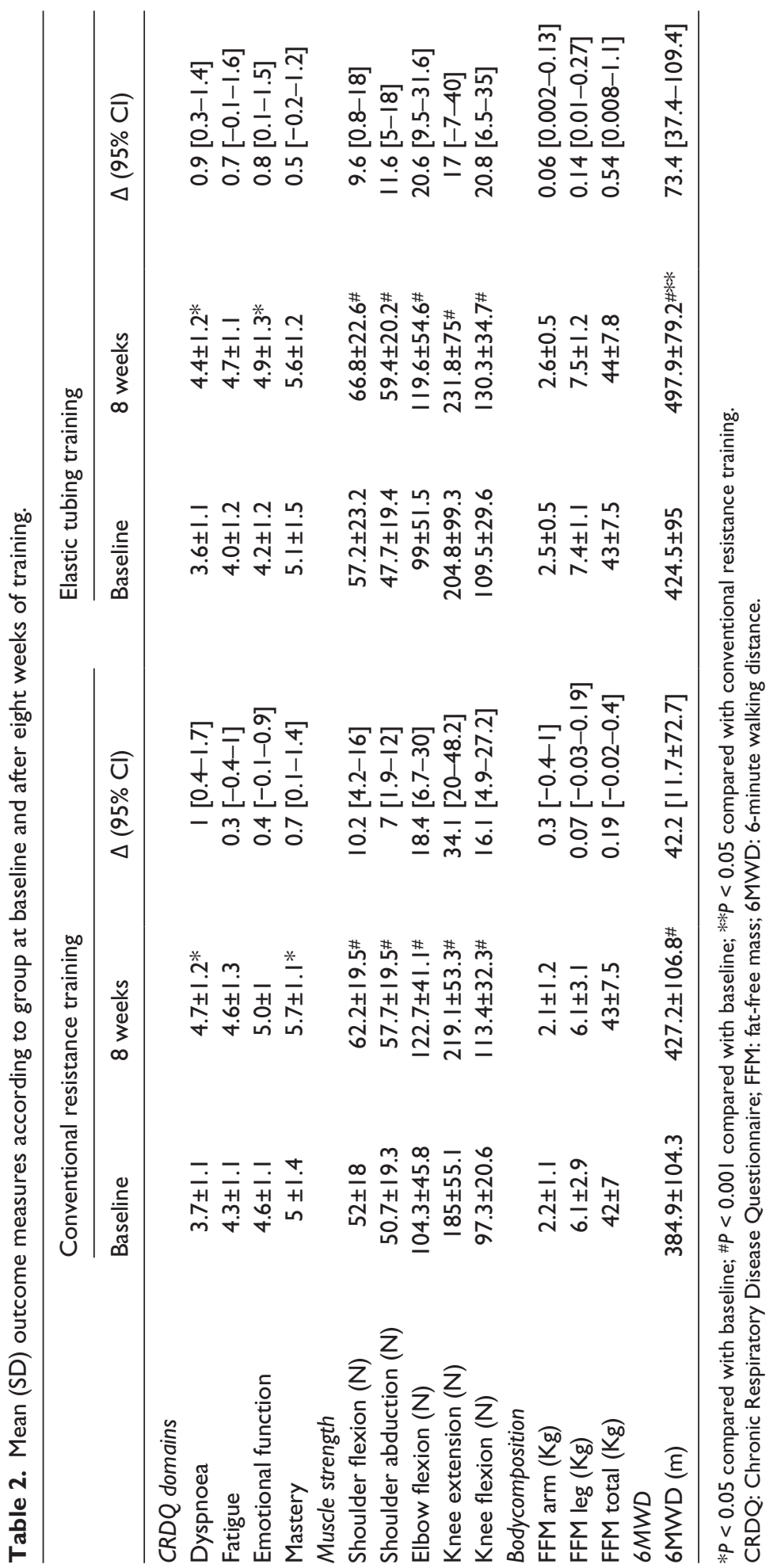




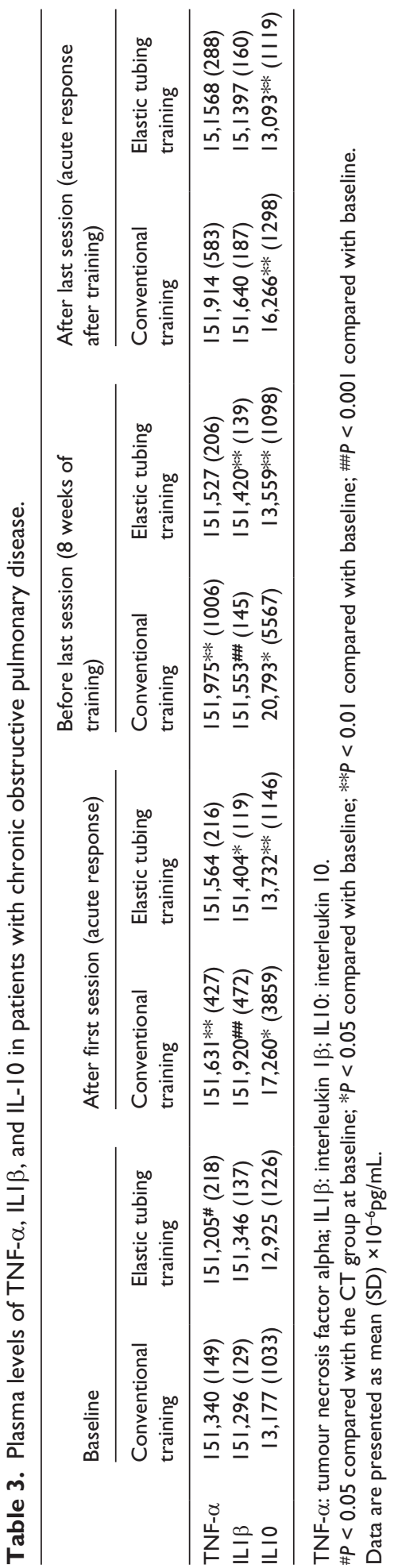

ETT in patients with chronic obstructive pulmonary disease. O'Shea et al. ${ }^{12}$ demonstrated modest improvements in strength in people with chronic obstructive pulmonary disease using elastic bands, with no reduction in physical and psychological impairment, following a home-based resistance training programme. However, in that study, only one session was conducted under the supervision of a physiotherapist, which may have resulted in lower exercise intensities. Furthermore, this study showed a low completion rate. In our study, patients were closely supervised by physiotherapists during the exercise protocol, and there were significant gains in upper and lower body strength after training. The distance achieved in the 6-minute walk test was positively correlated with quadriceps force in both groups at baseline. CT showed smaller differences in strength gain compared with the findings obtained by Spruit et al., ${ }^{18}$ although they used a 12 -week training programme.

In our study, both protocols improved functional exercise capacity after training, but the ETT group showed a larger gain in the 6-minute walking distance compared with the CT group. In addition to improved strength, elastic tubing has also been used to improve proprioception and postural control during joint rehabilitation, which could have contributed to the greater improvement in the functional exercise capacity observed in this group. The CT group showed less improvement in the 6-minute walking distance compared with the findings from Spruit et al. ${ }^{18}$ However, the differences in the population profile between studies should be taken into account. Brazilians have a longer 6-minute walking distance compared with other populations. ${ }^{28}$ In our study, patients with moderate to severe chronic obstructive pulmonary disease presented 6-minute walking distances at baseline that were $83 \%$ of the predicted distances (almost half of that study). ${ }^{18}$ After training, the conventional and elastic tubing groups reached an average of $93 \%$ of the predicted 6-minute walking distance, which indicates the effectiveness of both protocols in improving functional exercise capacity.

Severe skeletal muscle loss has been related to poor health-related quality of life, reduced exercise capacity, and increased risk of death in chronic 
obstructive pulmonary disease patients. ${ }^{29-32}$ Both protocols produced improvements exceeding the minimum clinically significant difference of 0.5 in almost all domains of the CRDQ; only the emotional function and fatigue domains did not reach the minimum clinically important difference after eight weeks of training in the conventional group. The excessive symptoms of dyspnoea and fatigue in chronic obstructive pulmonary disease patients are partly caused by muscle weakness. ${ }^{11,30}$ Our patients demonstrated greater peripheral muscle strength after training that positively affected their dyspnoea and fatigue perception, as described previously. ${ }^{18,31-33}$ The improvement in the fatigue and dyspnoea domains was positively correlated in both groups, which may reflect improvements in exercise tolerance and the ability to perform daily activities.

Resistance training programmes can increase the fat-free mass in chronic obstructive pulmonary disease patients. ${ }^{29}$ In our study, there was a trend favouring improvement in fat-free mass after training in both groups. This finding should be interpreted carefully, as it was likely caused by a type II error. Further studies with a larger number of participants and longer training periods will help to confirm these findings.

Increased cytokine levels have been reported to have a causal relationship with peripheral muscle dysfunction, and interventions that promote the strengthening of large muscle groups may change these levels in elderly individuals. ${ }^{13}$ Evidence regarding the relationship between cytokine levels and resistance training is limited, because the majority of studies involving cytokines are related to endurance exercise training. Training protocols can also influence the responses to cytokines and result in different adaptations to exercise. ${ }^{34} \mathrm{In}$ healthy subjects, a single session of resistance training can increase plasma cytokines, and training adaptation may result in lower levels of these proinflammatory cytokines in response to exercise. ${ }^{33,34}$ However, previous studies concluded that physical exercise training does not have an anti-inflammatory effect in chronic obstructive pulmonary disease. ${ }^{10,35}$ This is the first study showing that a single session and 24 sessions of conventional resistance exercise and elastic tubing exercise training increased IL-1 $\beta$, followed by an increase in IL-10, as a regulatory mechanism. As described previously, IL-10 released in response to eccentric exercise leads to an anti-inflammatory response via inhibition of the production of IL-1 $\beta$ and TNF$\alpha .{ }^{36,37} \mathrm{TNF}-\alpha$ increased only in the conventional resistance group. Macrophages can release TNF- $\alpha$ in response to exercise. However, performance of exercise is related to the levels of inflammatory mediators. ${ }^{38}$ Therefore, it is reasonable to suggest that the elastic tubing protocol attenuated inflammatory markers and tissue damage compared with CT, which may have been owing to the smaller trained muscle mass (single leg/arm). However, future studies should be performed to clarify this hypothesis.

The limitation of the current study was mainly the limited number of patients with COPD from a specific geographical location. Therefore, a multicentre study is required to confirm these results. Additionally, a home-based resistance training programme with elastic tubing in these patients warrants investigation.

In summary, the present randomized trial suggests that ETT can play an important role in the clinical management of patients with moderate or severe chronic obstructive pulmonary disease by improving functional exercise capacity, muscle strength, and quality of life (as well as promoting exercise adaptation, as demonstrated by cytokine behaviour). Our study presents an alternative protocol for resistance training in the lower and upper limbs in patients with chronic obstructive pulmonary disease. In Brazil, the public health system has a very small number of services that offer resistance training programmes to chronic obstructive pulmonary disease patients owing to the high cost of the equipment (weight machines). Elastic tubing meets the needs of these services owing to its low cost and applicability, as demonstrated by this study, and may yield better outcomes in this patient population. In addition, the option of training with elastic tubing can also be extended to home treatment, which contributes to the maintenance of functional gains from supervised clinical training and health services and consequently reduces healthcare costs. 


\section{Clinical messages}

- In patients with chronic obstructive pulmonary disease, moderate-intensity resistance training programme using elastic tubing yielded greater improvement in functional exercise capacity compared with conventional training performed with weight machines.

- Elastic tubing-based resistance training has an equivalent effect on muscle strength and quality of life.

\section{Acknowledgements}

The authors express their thanks to Sergio Moikawa from FCT/UNESP for his statistical advice and guidance during the development of this research.

\section{Conflict of interest}

The authors declare that there are no conflicts of interest.

\section{Funding}

This research received no specific grant from any funding agency in the public, commercial, or not-for-profit sectors.

\section{References}

1. Richardson RS, Leek BT, Gavin TP, et al. Reduced mechanical efficiency in chronic obstructive pulmonary disease but normal peak VO2 with small muscle mass exercise. Am J Respir Crit Care Med 2004; 169(1): 89-96.

2. Bernard S, Whittom F, LeBlanc P, et al. Aerobic and strength training in patients with chronic obstructive pulmonary disease. Am J Respir Crit Care Med 1999; 159: 896-901.

3. Simpson K, Killian K, McCartney N, Stubbing DG and Jones NL. Randomised controlled trial of weight lifting exercise in patients with chronic airflow limitation. Thorax 1992; 47: 70-75.

4. Clark CJ, Cochrane LM, Mackay E and Paton B. Skeletal muscle strength and endurance in patients with mild COPD and the effects of weight training. Eur Respir $J$ 2000; 15: 92-97.

5. Spruit MA, Gosselink R, Troosters T, et al. Resistance versus endurance training in patients with COPD and peripheral muscle weakness. EurRespir $J$ 2002; 19: 1072-1078.
6. Langer D, Hendriks EJM, Burtin C, et al. A clinical practice guideline for physiotherapists treating patients with chronic obstructive pulmonary disease based on a systematic review of available evidence. Clin Rehabil 2009; 23(5): 445-462.

7. Greiwe JS, Cheng B, Rubin DC, et al. Resistance exercise decreases skeletal muscle tumour necrosis factor alpha in frail elderly humans. FASEB J 2001; 15: 475-482.

8. Petersen AMW and Pedersen BK. The anti-inflammatory effect of exercise. J Appl Physiol 2005; 98: 1154-1162.

9. Bruunsgaard H, Pedersen M and Pedersen BK. Aging and proinflammatory cytokines. Curr Opin Hematol 2001; 8: 131-136.

10. Vlist VD and Janssen TWJ. The potential anti-inflammatory effect of exercise in chronic obstructive pulmonary disease. Respiration 2010; 79: 160-174.

11. Rovny D. The scientific and clinical application of elastic resistance. Phys Ther 2004; 84(1): 109.

12. O'Shea SD, Taylor NF and Paratz JD. A predominantly home-based progressive resistance exercise program increases knee extensor strength in the short-term in people with chronic obstructive pulmonary disease: A randomised controlled trial. Aust J Physiother 2007; 53: 229-237.

13. Nyberg A, Lindstrom B and Wadell K. Assessing the effect of high-repetitive single limb exercises (HRSLE) on exercise capacity and quality of life in patients with chronic obstructive pulmonary disease (COPD): study protocol for randomized controlled trial. Trials 2012; 13: 114.

14. Bjørgen S, Hoff J, Husby VS, et al. Aerobic high intensity one and two legs interval cycling in chronic obstructive pulmonary disease: the sum of the parts is greater than the whole. Eur J Appl Physiol 2009; 106(4): 501-507.

15. Brønstad E, Dedichen H, Kirkeby-Garstad I, Wisløff U and Steinshamn S. Effects of one-leg exercise training on mitochondrial function in COPD patients. Clin Respir $J$ 2011; 5: 9-10.

16. GOLD Scientific Committee: Global strategy for the diagnosis, management and prevention of chronic obstructive pulmonary disease. http://www.goldcopd.org/(accessed 7 March 2014)

17. Troosters T, Gosselink R and Decramer M. Short and long-term effects of outpatient rehabilitation in patients with chronic obstructive pulmonary disease: A randomized trial. Am J Med 2000; 109: 207-212.

18. Spruit MA, Gosselink R, Troosters T, et al. Resistance versus endurance training in patients with COPD and peripheral muscle weakness. Eur Respir $J$ 2002; 19: 1072-1078.

19. O'Shea SD, Taylor NF and Paratz J. Peripheral muscle strength training in COPD: A systematic review. Chest 2004; 126(3): 903-914.

20. Miller MR, Crapo R, Hankinson J, et al. Standardisation of spirometry "ATS/ERS task force: Standardisation of lung function testing”. Eur Resp J 2005; 26: 319-338. 
21. Duarte AA, Pereira CAC and Rodrigues SC. Validation of new Brazilian predicted values for forced spirometry in Caucasians and comparison with predicted values obtained using other reference equations. J Bras Pneumol 2007; 33(5): 527-535.

22. ATS statement: guidelines for the six-minute walk test. ATS Committee on Proficiency Standards for Clinical Pulmonary Function Laboratories. Am J Respir Crit Care Med 2002; 166(1): 111-117.

23. Enright PL and Sherrill DL. Reference equations for the six minute walk in healthy adults. Am J Respir Crit Care Med 1998; 158: 1384-1387.

24. Guyatt GH, Berman LB, Townsend M, et al. A measure of quality of life for clinical trials in chronic lung disease. Thorax 1987; 47: 773-778.

25. Jaeschke R, Singer J and Guyatt GH. Measurement of health status. Ascertaining the minimal clinically important difference. Controlled Clin Trials 1989; 10: 407-415.

26. Li C, Ford ES, Zhao G, et al. Estimates of body composition with dual-energy X-ray absorptiometry in adults. $\mathrm{Am}$ J Clin Nutr 2009; 90: 1457-1465.

27. Lacasse Y, Wong E, Guyatt GH, King D, Cook DJ and Goldstein RS. Meta-analysis of respiratory rehabilitation in chronic obstructive pulmonary disease. Lancet 1996; 348: 1115-1119.

28. Casanova C, Celli BR, Barria P, et al. The 6-min walk distance in healthy subjects: reference standards from seven countries. Eur Respir J 2011; 37: 150-156.

29. Schols AM, Broekhuizen R, Weling-Scheepers CA, et al. Body composition and mortality in chronic obstructive pulmonary disease. Am J Clin Nutr 2005; 82: 53-59.
30. Hamilton AL, Killian KJ, Summers E, et al. Muscle strength, symptom intensity, and exercise capacity in patients with cardiorespiratory disorders. Am J Respir Crit Care Med 1995; 152(6): 2021-2031.

31. Gosselink R, Troosters T and Decramer M. Peripheral muscle weakness contributes to exercise limitation in COPD. Am J Respir Crit Care Med 1996; 153: 976980.

32. Mador MJ and Bozkanat E. Skeletal muscle dysfunction in chronic obstructive pulmonary disease. Respir Rev 2001; 2: 216-24.

33. Mostert R, Goris A, Weling-Scheepers C, et al. Tissue depletion and health related quality of life in patients with chronic obstructive pulmonary disease. Respir Med 2000; 94: 859-867.

34. Calle M and Fernandez ML. Effects of resistance training on the inflammatory response. Nutrition Res Pract 2010; 4(4): 259-269.

35. Bolton CE, Broekhuizen R, Ionescu AA, et al. Cellular protein breakdown and systemic inflammation are unaffected by pulmonary rehabilitation in COPD. Thorax 2007; 62: 109-114.

36. Petersen AMW and Pedersen BK. The antiinflammatory effect of exercise. J Appl Physiol 2005, 98: 1154-1162.

37. Pedersen BK and Febraio MA. Muscle as an endocrine organ: Focus on muscle-derived Interleukin-6. Physiol Rev 2008; 88: 1379-1406.

38. Steinacker JM, Lormes W, Reissnecker S, et al. New Aspects of the hormone and cytokine response to training. Eur J Appl Physiol 2004; 91: 382-391. 